\title{
Real-World Data of Pyrotinib-Based Therapy in Metastatic HER2-Positive Breast Cancer: Promising Efficacy in Lapatinib-Treated Patients and in Brain Metastasis
}

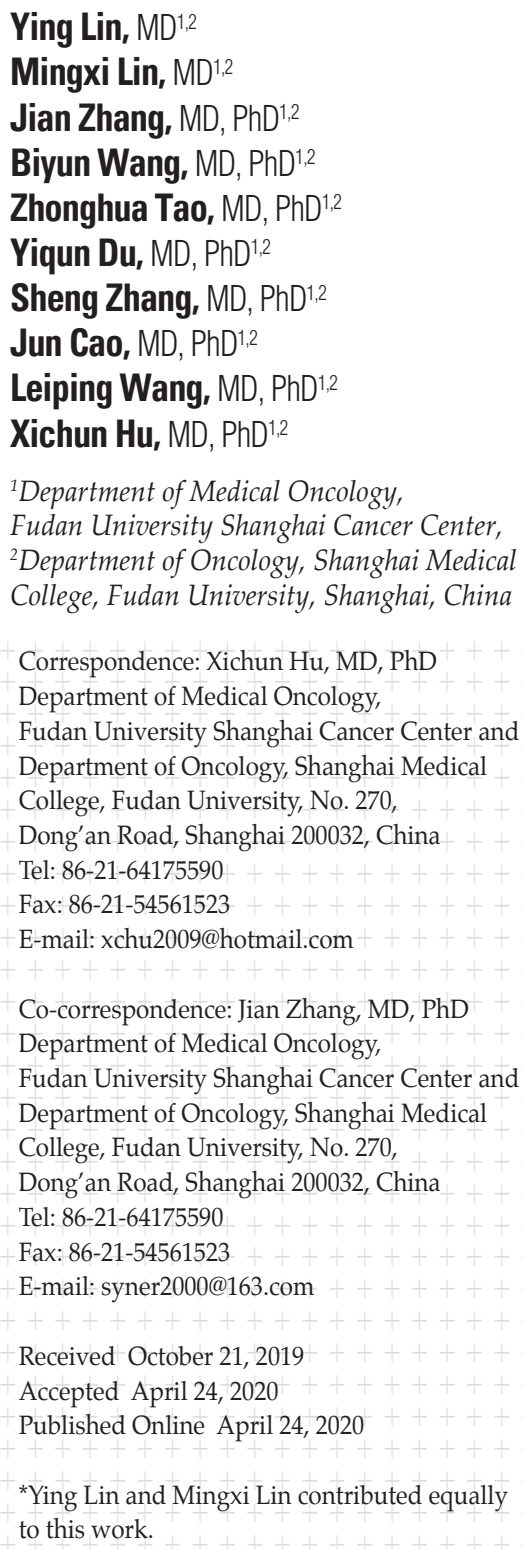

\begin{abstract}
Purpose
Pyrotinib is a newly-developed irreversible pan-ErbB receptor tyrosine kinase inhibitor. This study reported the first real-world data of pyrotinib-based therapy in metastatic human epidermal growth factor receptor 2 (HER2)-positive breast cancer (BC), focusing on efficacy in lapatinib-treated patients and in brain metastasis.
\end{abstract}

\section{Materials and Methods}

One hundred thirteen patients with metastatic HER2-positive BC treated with pyrotinibbased therapy in Fudan University Shanghai Cancer Center under non-clinical trial settings from September 1, 2018 to March 1, 2019 were included.

\section{Results}

Over half patients have received more than two lines of systematic therapy and exposed to two or more kinds of anti-HER2 agents. Most patients received a combined therapy, commonly of pyrotinib plus capecitabine, or vinorelbine or trastuzumab. Median progressionfree survival (PFS) was 6.3 months (range, 5.54 to 7.06 months) and objective response rate (ORR) was $29.5 \%$, with two patients (1.9\%) achieving complete response. Lapatinibnaïve patients had significantly longer PFS than lapatinib-treated patients ( 9.0 months vs. 5.4 months, $p=0.001$ ). ORR for lapatinib-treated patients was $23.2 \%$. Thirty-one of 113 patients have brain metastasis. Median PFS was 6.7 months and intracranial ORR was $28 \%$. For patients without concurrent radiotherapy and/or brain surgery, the ORR was very low (6.3\%). But for patients receiving concurrent radiotherapy and/or brain surgery, the ORR was $66.7 \%$, and three patients achieved complete response. Most common adverse event was diarrhea.

\section{Conclusion}

Pyrotinib-based therapy demonstrated promising effects in metastatic HER2-positive BC and showed activity in lapatinib-treated patients. For patients with brain metastasis, pyrotinib-based regimen without radiotherapy showed limited efficacy, but when combined with radiotherapy it showed promising intracranial control.

\section{Key words}

Pyrotinib, HER2-positive breast cancer, Tyrosine kinase inhibitor, Lapatinib-treated, Brain metastasis

\section{Introduction}

Human epidermal growth factor receptor 2 (HER2)-positive breast cancer (BC) consists of $15 \%-20 \%$ of BC [1]. Before the era of HER2-targeted therapy, HER2-positive BC was aggressive, easily recurrent and had poor prognosis [1]. The development of anti-HER2 therapy has dramatically improved the survival of this BC subtype [1].

Recently pyrotinib, a novel oral pan-ErbB receptor tyrosine kinase inhibitor (TKI), has shown very promising results in 
metastatic HER2-positive BC [2-5]. In a phase II study, pyrotinib plus capecitabine had significantly higher objective response rate (ORR) $(78.5 \%$ vs. $57.1 \%, \mathrm{p}=0.01)$ and longer progression-free survival (PFS; 18.1 months vs. 7.0 months, $\mathrm{p}<$ 0.001) compared to lapatinib plus capecitabine [5]. Recently, PHENIX study, a double-blinded, multicenter, randomized phase III study, showed that pyrotinib plus capecitabine significantly prolonged PFS (11.1 months vs. 4.1 months, $\mathrm{p}<$ $0.001)$ and increased ORR $(68.6 \%$ vs. $16.0 \%, p<0.001)$ than capetabine monotherapy [2]. Both studies included patients with metastatic HER2-positive BC previously treated with no more than two lines of systematic therapy. Pyrotinib was approved in China in August 2018 for metastatic HER2-positive $\mathrm{BC}$ because of the remarkable result of the above phase II study and is currently in phase I clinical trial in the United States.

Pyrotinib and neratinib are both irreversible ErbB receptor TKIs, which are different in nature from lapatinib, a reversible HER1 and HER2 receptor TKI. Both pyrotinib and neratinib were found to have superior efficacy than lapatinib $[5,6]$. The median PFS of 11.1 months in PHENIX study achieved by pyrotinib plus capecitabine is comparable to that of 8.8 months achieved by neratinib plus capetabine as third or later line therapy in NALA trial and that of 12.9 months achieved by neratinib plus paclitaxel as first-line treatment in the NEfERT$\mathrm{T}$ trial [6,7], suggesting the potentially comparable efficacy of pyrotinib to neratinib. However, a common problem in both the phase II and phase III studies of pyrotinib is that patients were not optimally treated with anti-HER2 therapy before trials, and some patients were even naïve to trastuzumab $[2,5]$. Therefore, neither study could fully represent the major populations of global metastatic HER2-positive BC patients, who were usually optimally treated with multiple anti-HER2 agents, especially in western countries where more drugs were available [8]. Questions about whether the results pf pyrotinib clinical trials were applicable in the current setting for anti-HER2 therapy remains [8].

Another question is whether pyrotinib is effective in patients with exposure to lapatinib. As for neratinib, in lapatinibtreated cohort of TBCRC022 trial, neratinib plus capetabine arm had intracranial ORR of $33 \%$, extracranial ORR of $43 \%$, and median PFS of 3.1 months, demonstrating the activity of neratinib in lapatinib-treated patients [9]. However, no data so far is available regarding the activity of pyrotinib in lapatinib-treated patient.

HER2-positive BC has higher incidence of brain metastasis than other BC subtypes, with a risk as high as 35\%-50\% $[10,11]$. Brain metastasis in BC is associated with very poor clinical outcome, with 1-year overall survival (OS) less than $50 \%$ [11]. Blood brain barrier (BBB) hinders the efficacy of many drugs because of the limited penetration. Anti-HER2 TKIs have been widely exploited due to their small molecule property that enhances the ability to penetrate the BBB [11].
Radiotherapy also is a common option for local control of brain lesions. Despite these efforts, the treatments for brain metastasis are still limited. In the subgroup analysis of PHENIX study, 31 patients with brain metastasis were further analyzed, pyrotinib plus capetabine prolonged PFS by 2.7 months compared to capetabine (6.9 months vs. 4.2 months, $\mathrm{p}=0.011$ ), showing promising efficacy in brain metastasis [2]. However, the sample size is small and more data is needed to verify the intracranial efficacy of pyrotinib.

This study aimed to evaluate the efficacy of pyrotinib-based therapy in metastatic HER2-positive BC in the real world, especially focusing on lapatinib-treated patients and on brain metastasis, and to explore the efficacy and safety when it is combined with agents other than capetabine. To our knowledge, this is the first real-world study of pyrotinib-based therapy, and first study evaluating the efficacy of pyrotinib in lapatinib-treated patients.

\section{Materials and Methods}

\section{Patient population and data collection}

Information of patients with metastatic HER2-positive BC treated with pyrotinib-based therapy in Fudan University Shanghai Cancer Center (FUSCC) under non-clinical trial settings from September 1, 2018 to March 1, 2019 was obtained. Eligible patients were women with histologically confirmed HER2-positive locally recurrent or metastatic BC. Patients who once received pyrotinib in clinical trial settings were excluded. For patients who underwent biopsies in the metastatic sites, hormone receptor and HER2 status were determined based on metastatic lesions. Last follow-up time was September 2019.

\section{Treatment and dose modification}

Patients were prescribed with pyrotinib in routine clinical practice. The standard dosage is $400 \mathrm{mg}$ single dose orally per day. Starting dose, dose modification, dose interruption, treatment discontinuation, combination therapy with cytotoxic drugs and/or anti-HER2 agents and/or radiotherapy were determined by physicians' choice based on previous clinical trials results, general health status and willing of patients.

\section{Efficacy and safety assessments}

Tumor response assessments were based on Response Evaluation Criteria in Solid Tumor (RECIST) criteria (ver. 1.1) using radiologic scans, including computed tomography (CT) or magnetic resonance imaging (MRI). Adverse events (AEs) were assessed according to the National Cancer Institute Common Terminology Criteria for Adverse Events (CT$\mathrm{CAE}, 4.03)$. AEs were collected based on a patient self-reporting system and by reviewing biochemical test results. 
The primary end point was PFS, which was defined as the time from initiating pyrotinib to date of disease progression confirmed by CT/MRI scan or death of any cause, regardless of whichever would occur first. Secondary endpoint included ORR, OS, and safety. The ORR was defined as the proportion of patients with complete response (CR) or partial response (PR). OS was defined as the time period from initiating pyrotinib treatment to the date of death of any cause. Disease-free interval was defined as the time from primary radical surgery to the date of relapse.

\section{Statistical analysis}

Median PFS and OS were calculated by the Kaplan-Meier method and the subgroup comparisons were evaluated using the log-rank test. Median follow-up period was calculated by reverse Kaplan-Meier method. Stepwise Cox regression model was used to analyze the correlations between factors and PFS. All statistical analyses were performed using SPSS ver. 19 (SPSS Inc., Chicago, IL). All statistical tests were twotailed and $\mathrm{p}<0.05$ was considered statistically significant.

\section{Ethical statement}

This study was approved by the FUSCC Ethics Committee (approval No. 2003215-19) and performed in accordance with the 1964 Helsinki declaration and its later amendments or comparable ethical standards. Informed consents were obtained in accordance with study protocol.

\section{Results}

\section{Baseline characteristics}

A total of 122 patients were prescribed with pyrotinib under non-clinical settings in FUSCC from September 1, 2018, to March 1, 2019. Nine patients were excluded because they transferred to other hospital and no further information can be accessed. Therefore, 113 patients were included in our study. Median follow-up duration was 8.4 months (interquartile range, 7.0 to 9.9 months). Baseline characteristics were summarized in Table 1. Median age of patients was 53.4 years (range, 24 to 84 years). Thirty-one patients $(27.4 \%)$ had brain metastasis. All patients except $1(99.1 \%)$ were prior exposed to anti-HER2 therapy, with $99.1 \%$ patients exposed to trastuzumab and $50.4 \%$ exposed to lapatinib (Table 1). Ninety-one out of the 113 patients had received primary radical surgery when first diagnosed. Of the 91 patients with primary surgery, $43(47.3 \%)$ have received standard 1-year adjuvant trastuzumab treatment, seven $(7.7 \%)$ had inadequate adjuvant trastuzumab therapy due to all kinds of reasons, seven $(7.7 \%)$ had primary resistance and relapsed during adjuvant trastuzumab therapy, and the remaining $34(37.4 \%)$ did not receive any anti-HER2 adjuvant therapy. Sixty-one point nine percent of patients received more than
Table 1. Patient characteristics at baseline

\begin{tabular}{|c|c|}
\hline Characteristic & No. $(\%)(n=113)$ \\
\hline Age, median (range, yr) & $53.4(24-84)$ \\
\hline \multicolumn{2}{|l|}{ HR status } \\
\hline HR positive & $45(39.8)$ \\
\hline HR negative & $68(60.2)$ \\
\hline \multicolumn{2}{|l|}{ ECOG performance status } \\
\hline $0-1$ & 107 (94.7) \\
\hline$\geq 2$ & $5(4.4)$ \\
\hline Unknown & $1(0.9)$ \\
\hline \multicolumn{2}{|l|}{ DFI } \\
\hline Primary metastatic & $22(19.5)$ \\
\hline $\mathrm{DFI} \leq 1 \mathrm{yr}$ & $16(14.2)$ \\
\hline $\mathrm{DFI}>1 \mathrm{yr}$ & $75(66.4)$ \\
\hline \multicolumn{2}{|l|}{ Metastatic sites } \\
\hline Lymph nodes & $74(65.5)$ \\
\hline Lung & $63(55.8)$ \\
\hline Liver & $57(50.4)$ \\
\hline Bone & $48(42.5)$ \\
\hline Brain & $31(27.4)$ \\
\hline Local recurrence & $30(26.5)$ \\
\hline Pleura & $21(18.6)$ \\
\hline Contralateral breast & $6(5.3)$ \\
\hline \multicolumn{2}{|l|}{ No. of metastatic sites } \\
\hline 1 & $25(22.1)$ \\
\hline 2 & $28(24.8)$ \\
\hline 3 & $19(16.8)$ \\
\hline$\geq 4$ & $41(36.3)$ \\
\hline \multicolumn{2}{|l|}{ Visceral metastases } \\
\hline Yes & $100(88.5)$ \\
\hline No & $13(11.5)$ \\
\hline \multicolumn{2}{|c|}{ Lines of systematic therapy of pyrotinib } \\
\hline 1 & $20(17.7)$ \\
\hline 2 & $23(20.4)$ \\
\hline 3 & $25(22.1)$ \\
\hline$\geq 4$ & $45(39.8)$ \\
\hline \multicolumn{2}{|c|}{ Prior HER2-targeted therapy } \\
\hline Trastuzumab & $112(99.1)$ \\
\hline Lapatinib & $57(50.4)$ \\
\hline T-DM1 & $12(10.6)$ \\
\hline Pertuzumab & $5(4.4)$ \\
\hline
\end{tabular}

HR, hormone receptor; ECOG, Eastern Cooperative Oncology Group; DFI, disease-free interval; HER2, human epidermal growth factor receptor 2 .

two lines of systematic therapy before. Fifty-three (46.9\%), 46 $(40.7 \%)$, and $14(12.4 \%)$ patients were exposed to 1,2 , and 3 kinds of anti-HER2 agents, respectively.

\section{Treatment administration}

Treatment administration was shown in Table 2. Most patients $(96.5 \%)$ received a combined therapy. Besides the combination of pyrotinib plus capetabine previously studied in 
Table 2. Treatment administration

\begin{tabular}{|lc|}
\hline Pyrotinib treatment & No. $(\%)(\mathbf{n}=\mathbf{1 1 3})$ \\
\hline Regimens & \\
\hline Single agent & $4(3.5)$ \\
\hline Combined therapy & \\
\hline Pyrotinib+capetabine & $67(59.3)$ \\
\hline Pyrotinib+trastuzumab+capetabine & $14(12.4)$ \\
\hline Pyrotinib+vinorelbine & $9(8.0)$ \\
\hline Pyrotinib+trastuzumab & $8(7.1)$ \\
\hline Pyrotinib+paclitaxel & $3(2.7)$ \\
\hline Other & $8(7.1)$ \\
\hline Dosage & \\
\hline Starting dosage $(\mathrm{mg} /$ day) & \\
\hline 160 & $1(0.9)$ \\
\hline 240 & $1(0.9)$ \\
\hline 320 & $3(2.6)$ \\
\hline 400 & $108(95.6)$ \\
\hline Dose escalation $(\mathrm{mg} /$ day) & \\
\hline $160 \rightarrow 400$ & $1(0.9)$ \\
\hline $240 \rightarrow 400$ & $1(0.9)$ \\
\hline $320 \rightarrow 400$ & $1(0.9)$ \\
\hline Dose reduction (mg/ day) & $24(21.2)$ \\
\hline $400 \rightarrow 320$ & $2(1.8)$ \\
\hline $400 \rightarrow 320 \rightarrow 240$ & $7(6.2)$ \\
\hline Treatment discontinuation due to AEs & \\
\hline
\end{tabular}

AEs, adverse events.

clinical trial, common combined agents also included vinorelbine and trastuzumab. Most patients started pyrotinib treatments at the standard dose of $400 \mathrm{mg} /$ day, but $26(23.0 \%)$ and $43(38.1 \%)$ patients experienced dose reduction and treatment interruption respectively. The most common AEs causing dose reduction and treatment interruption were diarrhea, vomiting, nausea, and anorexia. Four patients were more than 70 years old, and they all started pyrotinib at standard dose of $400 \mathrm{mg}$. One out of four experienced dose reduction twice, and another one out of four experienced dose reduction once. Seven patients (6.2\%) discontinued treatment permanently due to intolerant AEs, including three due to diarrhea, three due to vomiting and one due to simultaneous diarrhea and vomiting.

\section{Efficacy in all patients}

A total of 113 patients were included in PFS analysis. Median PFS was 6.3 months (range, 5.54 to 7.06 months) (Fig. 1A). Forty patients (35.4\%) were still in treatment and median OS has not achieved by the time of this study.

A total of 105 patients were included in ORR analysis, with eight patients excluded because of lack of measurable lesions (Table 3). ORR was $29.5 \%$, with $2(1.9 \%)$ patients achieving CR. Of the two patients with CR response, one had a pri-
A

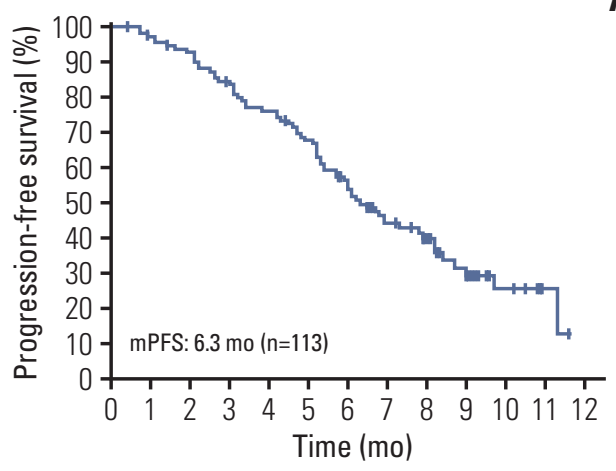

B

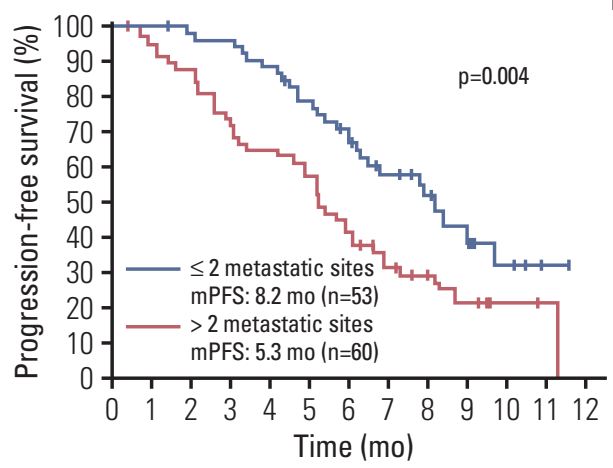

C

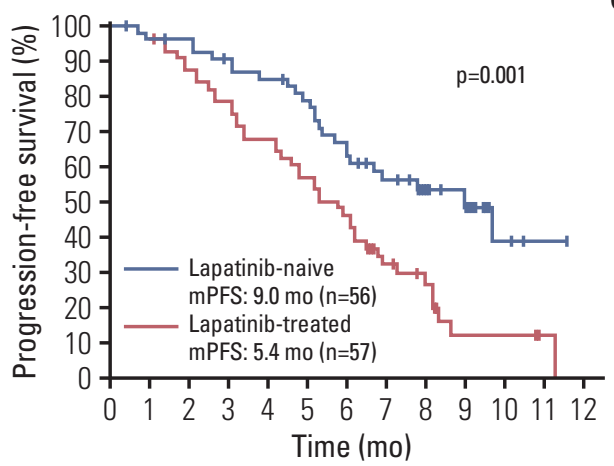

Fig. 1. Kaplan-Meier plot of progression-free survival and logrank analysis of predictors of pyrotinib-based treatment. (A) Kaplan-Meier plot of progression-free survival of all patients treated with pyrotinib-based treatment. (B) Kaplan-Meier plot of progression-free survival for patients with $\leq 2$ metastatic sites and $>2$ metastatic sites. (C) Kaplan-Meier plot of progressionfree survival for patients with and without prior lapatinib exposure. $\mathrm{mPFS}$, median progression-free survival.

mary stage IV disease who has not been exposed to any antiHER2 therapy and received pyrotinib plus trastuzumab plus docetaxel as first-line therapy. The other patient had a tumor with primary resistance to trastuzumab who experienced metastasis to brain during perioperative systematic therapy and received pyrotinib plus capetabine plus whole brain radiotherapy as first-line therapy. 
Table 3. ORR rate in all patients and in patients with prior exposure to lapatinib

\begin{tabular}{|lc|}
\hline Response & No. (\%) \\
\hline All patients & 105 \\
\hline Complete response & $2(1.9)$ \\
\hline Partial response & $29(27.6)$ \\
\hline Stable disease & $44(41.9)$ \\
\hline Progressive disease & $22(21.0)$ \\
\hline No data & $8(7.6)$ \\
\hline ORR & $31(29.5)$ \\
\hline Lapatinib-treated patients & 56 \\
\hline Complete response & 0 \\
\hline Partial response & $13(23.2)$ \\
\hline Stable disease & $26(46.4)$ \\
\hline Progressive disease & $16(28.6)$ \\
\hline No data & $1(1.8)$ \\
\hline ORR & $13(23.2)$ \\
\hline
\end{tabular}

ORR, objective response rate.

The number of metastatic sites ( $\leq 2$ vs. $>2$ ) and prior exposure to lapatinib were significantly correlated with PFS in log-rank analysis ( $\mathrm{p}=0.004$ and $\mathrm{p}=0.001$, respectively) (Fig. $1 \mathrm{~B}$ and $\mathrm{C})$, and were independent predictors of PFS in Cox multivariate analysis $(\mathrm{p}=0.048$ and $\mathrm{p}=0.002$, respectively)
(Table 4). Patients exposed to one kind of anti-HER2 agent had significantly longer PFS (9.0 months) than those exposed to 2 (5.9 months) or 3 (5.1 months) kinds of anti-HER2 agents (S1A Fig.).

\section{Efficacy of pyrotinib-based therapy in lapatinib-treated patients}

Fifty-seven patients were previously exposed to lapatinib and later received pyrotinib-based therapy. One patient lacked measurable lesions. Of the remaining 56 patients, 23.2\% achieved PR response and no one achieved CR response (Table 3). Median PFS in patients with and without previous exposure to lapatinib were 5.4 months and 9.0 months, respectively ( $\mathrm{p}=0.001$ ) (Fig. 1C).

\section{Efficacy of pyrotinib-based therapy in brain metastasis}

Thirty-one patients had brain metastasis at baseline. There is no difference in PFS between patients with and without brain metastasis ( $p=0.696)$ (S1B Fig.). Fifty-four point eight percent of patients have received radiotherapy of brain lesions in the previous recurrence. Overall median PFS (intracranial and extracranial lesions considered) for patients with brain metastasis was 6.7 months (range, 4.69 to 8.71 months). By the time of the study, 10 patients were still undergoing treatment.

Table 4. Log-rank and Cox multivariate analysis of factors associated with progression-free survival

\begin{tabular}{|c|c|c|c|}
\hline \multirow{2}{*}{ Characteristic } & \multirow{2}{*}{$\begin{array}{l}\text { Log-rank analysis } \\
\text { p-value }\end{array}$} & \multicolumn{2}{|c|}{ Cox multivariate analysis } \\
\hline & & $\overline{\text { p-value }}$ & Hazard ratio $(95 \% \mathrm{CI})$ \\
\hline DFI (> 1 yr vs. $\leq 1$ yr vs. primary stage IV) & 0.510 & 0.075 & \\
\hline$>1 \mathrm{yr} v \mathrm{~s} . \leq 1 \mathrm{yr}$ & & & $2.212(1.079-4.535)$ \\
\hline$>1$ yr vs. primary stage IV & & & $0.887(0.477-1.650)$ \\
\hline Types of metastasis (non-visceral vs. visceral) & 0.428 & 0.890 & $1.064(0.441-2.571)$ \\
\hline No. of metastatic sites ( $\leq 2$ vs. $>2$ ) & 0.004 & 0.048 & $1.778(1.005-3.145)$ \\
\hline Prior exposure to lapatinib (no vs. yes) & 0.001 & 0.002 & $2.313(1.347-3.971)$ \\
\hline HR status (HR+ vs. HR-) & 0.145 & 0.552 & $1.174(0.692-1.992)$ \\
\hline Age group ( $>60 \mathrm{yr}$ vs. $\leq 60 \mathrm{yr}$ ) & 0.556 & 0.948 & $1.018(0.593-1.749)$ \\
\hline
\end{tabular}

$\mathrm{CI}$, confidence interval; DFI, disease free interval; HR, hormone receptor.

Table 5. Objective response rate for brain lesions

\begin{tabular}{lccc} 
Response & $\begin{array}{c}\text { All patients } \\
(\mathbf{n}=\mathbf{2 5})\end{array}$ & $\begin{array}{c}\text { Patients without concurrent } \\
\text { radiotherapy or surgery (n=16) }\end{array}$ & $\begin{array}{c}\text { Patients with concurrent } \\
\text { radiotherapy and/or surgery }(\mathbf{n = 9 )}\end{array}$ \\
Best response & $3(12.0)$ & 0 & $3(33.3)$ \\
$\quad$ Complete response & $4(16.0)$ & $1(6.3)$ & $3(33.3)$ \\
Partial response & $9(36.0)$ & $8(50.0)$ & $1(11.1)$ \\
Stable disease & $5(20.0)$ & $5(31.3)$ & 0 \\
Progressive disease & $5(20.0)$ & $2(12.5)$ & $3(33.3)$ \\
No data & $7(28.0)$ & $1(6.3)$ & $6(66.7)$ \\
\hline ORR &
\end{tabular}

Values are presented as number $(\%)$. ORR, objective response rate. 
Table 6. Grade 3 to 4 adverse events

\begin{tabular}{lc} 
Grade 3 to 4 adverse events & No. of patients $(\%)$ \\
\hline Diarrhea & $30(26.5)$ \\
\hline PPES & $11(9.7)$ \\
Neutropenia & $5(4.4)$ \\
\hline Elevated aminotransferase & $4(3.5)$ \\
Anemia & $4(3.5)$ \\
Vomit & $4(3.5)$ \\
Leukopenia & $3(2.7)$ \\
Weight loss & $2(1.8)$ \\
Thrombocytopenia & $2(1.8)$ \\
\hline Mucositis oral & $1(0.9)$ \\
Fatigue & $1(0.9)$ \\
\hline Anorexia & $1(0.9)$ \\
Blood bilirubin increased & $1(0.9)$ \\
\hline
\end{tabular}

PPES, palmar-plantar erythrodysesthesia syndrome.

Twenty-five patients were included in the intracranial ORR analysis, with six patients excluded due to lack of measurable brain lesions (Table 5). Sixteen out of 25 patients did not receive concurrent radiotherapy or surgery of brain (Table 5). ORR was $28 \%$, with $3 / 25(12 \%)$ patients achieving CR and 4/ $25(16 \%)$ patients achieving PR (Table 5). The three patients with $\mathrm{CR}$ response all received pyrotinib plus capetabine plus radiotherapy, and one of them has been exposed to three lines of systematic therapy before. In patients receiving pyrotinib-based systematic therapy and concurrent radiotherapy (8 patients) and / or surgery (1 patient) of the brain, the ORR was as high as $66.7 \%$ (6/9) (Table 5). After excluding those combined with radiotherapy, only $1 / 16(6.3 \%)$ patients achieved PR and no patient achieved CR (Table 5).

\section{Safety}

As we used a patient self-reporting system to document AEs, and given the retrospective nature of the study, omission in reporting AEs was unavoidable. Here we report the grade 3 to $4 \mathrm{AEs}$ (Table 6). The most common grade 3 to $4 \mathrm{AEs}$ were diarrhea $(26.5 \%)$, palmar-plantar erythrodysesthesia syndrome (PPES, 9.7\%), neutropenia (4.4\%). Excluding those with pyrotinib monotherapy and those combined with capetabine, toxicities remained tolerable. Most common grade 3 to 4 AEs were diarrhea (38.1\%) and neutropenia (4.8\%).

\section{Discussion}

The advent of HER2 targeted therapy has dramatically improved the prognosis of HER2-positive BC. Pyrotinib is a novel anti-HER2 TKI recently approved in China. Our study showed promising effects of pyrotinib-based therapy with a median PFS of 6.3 months and an ORR of $29.5 \%$ in metastatic
HER2-positive BC. Comparing to the median PFS of 18.1 months and 11.1 months and the ORR of $78.5 \%$ and $68.6 \%$ achieved by pyrotinib plus capecitabine combination in previous phase II and III trials [2,5], our data were less fascinating. Several reasons should be taken into consideration. First of all, previous clinical trials included patients treated with two or less lines and some patients have not been exposed to any anti-HER2 therapy [2,5]. But in our cohort, over half patients were treated with more than two lines of systematic therapy, and over half received two or more kinds of antiHER2 agents. Therefore, our cohort represented a treatment refractory population, and also the general population of patients with metastatic HER2-positive BC who were usually heavily treated with multiple anti-HER2 agents. Results of our study provided more experience outside the clinical trials for clinicians in treating general metastatic HER2-positive BC patients. Secondly, the follow-up time of our study is rather short and more than $30 \%$ of patients were still in treatment. However, we should also keep in mind that our study cohort included few patients previously exposed to pertuzumab and/or T-DM1. Pertuzumab and/or T-DM1 were common choices for front line treatments of HER2-positive BC patients globally. However, in China, pertuzumab was newly-approved and T-DM1 is waiting to be approved, which limited their usage in Chinese patients. Therefore, the role of pyrotinib in more heavily treated patients needs further global study. We hope the result from the phase I clinical trial of pyrotinib in the United States of America might shed some light on this question.

The efficacy of pyrotinib-based therapy was significantly better in lapatinib-naïve patients than in lapatinib-treated patients (Fig. 1C). In lapatinib-naïve group, pyrotinib-based therapy achieved a median PFS of 9.0 months, numerically comparable to that of neratinib plus capetabine arm (8.8 months) and better than that of lapatinib plus capetabine arm (6.6 months) in NALA study. In lapatinib-treated group, pyrotinib-based therapy had an ORR of $23.2 \%$, which was a bit less than those from TBCRC022 trial (intracranial ORR $33 \%$ and extracranial ORR of $43 \%$ ), but the median PFS of 5.4 months was numerically better than that from TBCRC022 trial (3.1 months). For the first time to our knowledge, this result provided evidence of the activity of pyrotinib-based therapy after failure of lapatinib-based treatment.

For HER2-positive BC, brain is always a sanctuary site [1]. For patients with brain metastasis, treatments remain limited and prognosis remains poor. Although anti-HER2 monoclonal antibodies and HER2-directed antibody drug conjugates were shown to improve survival in patients with brain metastasis in several studies [12-14], their intracranial effects remain controversial due to large-molecule property that hinders the infiltration through BBB. Anti-HER2 TKIs are important treatment options for brain metastasis. Petrelli et al. [15] conducted a pooled analysis including 12 studies 
of the efficacy of lapatinib plus capecitabine in brain metastasis in HER2-positive BC. Results showed that lapatinib plus capecitabine achieved an ORR of nearly $30 \%$ and a median PFS of 4.1 months [15]. In the TBCRC022 trial, neratinib plus capecitabine has also shown promising efficacy in HER2positive BC with brain metastasis with an ORR of $49 \%$ and $33 \%$ and median PFS of 5.5 and 3.1 months in lapatinib-naïve and lapatinib-treated patients respectively [9].

In our study, in patients with brain metastasis and only received pyrotinib-based therapy without local control of brain metastasis such as radiotherapy and surgery, the ORR was very low $(6.3 \%)$, which was disappointing when compared to the efficacy of neratinib plus capetabine in brain metastasis in TBCRC022 trial. More data are needed to further evaluate the intracranial efficacy of pyrotinib. However, in patients combined pyrotinib-based systematic therapy with radiotherapy and/ or surgery, the ORR was as high as $66.7 \%$, and three out of nine patients achieved CR in brain lesions. This response rate was much higher than the previous study of lapatinib plus concurrent radiotherapy, which reported an ORR of $35 \%$, in the treatment of brain metastasis [16], suggesting a possible treatment regimen of pyrotinib plus cytotoxic drugs plus radiotherapy for better intracranial control.

Pyrotinib-based therapy was generally well-tolerated. The most common grade 3 to 4 AEs was diarrhea, which was consistent with reports of the previous clinical trial. No severe $\mathrm{AE}$ was reported. Grade 3 to 4 PPES was less than that reported in clinical trials [2,5], mainly because about $40 \%$ of patients did not receive capecitabine as combined therapy. Combinations with agents other than capecitabine also demonstrated good safety profile, suggesting more combination options including anti-HER2 antibodies and cytotoxic drugs.
However, given that a patient self-reporting system is used in reporting AEs and the retrospective nature of the study, oblivion in AE reporting was unavoidable.

Pyrotinib combination therapy demonstrated promising effects in metastatic HER2-positive BC with tolerable side effects, especially in lapatinib-naïve patients, and also some activity in lapatinib-treated patients. However, efficacy of pyrotinib-based therapy without concurrent radiotherapy in brain metastasis was not satisfying in our study and more investigations are needed in the future. But when combined with radiotherapy, pyrotinib-based therapy demonstrated remarkable intracranial disease control. More clinical trials are needed to further exploit the potential of this novel irreversible pan-ErbB receptor TKI.

\section{Electronic Supplementary Material}

Supplementary materials are available at Cancer Research and Treatment website (https:// www.e-crt.org).

\section{Conflicts of Interest}

Conflicts of interest relevant to this article was not reported.

\section{Acknowledgments}

This study was supported by the Shanghai Municipal Science and Technology Commission Guidance Project, P.R. China (Contract No. 18411967800); Shanghai Municipal Commission of Health and Family Planning (Grant No. 201640069); Shanghai Natural Science Foundation (Grant No. 17ZR1405700); Research grant from Shanghai Hospital Development Center (Grant No. SHDC12018X03); and Shanghai Anticancer Association EYAS Project (Grant No. SACACY1B03). The authors would like to thank all the doctors, nurses, patients and their family members for their supports to our study.

\section{References}

1. Waks AG, Winer EP. Breast cancer treatment: a review. JAMA. 2019;321:288-300.

2. Jiang Z, Yan M, Hu X, Zhang Q, Ouyang Q, Feng J, et al. Pyrotinib combined with capecitabine in women with HER2+ metastatic breast cancer previously treated with trastuzumab and taxanes: a randomized phase III study. J Clin Oncol. 2019; 37(15 Suppl):1001.

3. Li X, Yang C, Wan H, Zhang G, Feng J, Zhang L, et al. Discovery and development of pyrotinib: a novel irreversible EGFR/ HER2 dual tyrosine kinase inhibitor with favorable safety profiles for the treatment of breast cancer. Eur J Pharm Sci. 2017; 110:51-61.

4. Ma F, Li Q, Chen S, Zhu W, Fan Y, Wang J, et al. Phase I study and biomarker analysis of pyrotinib, a novel irreversible pan-ErbB receptor tyrosine kinase inhibitor, in patients with human epidermal growth factor receptor 2-positive metastatic breast cancer. J Clin Oncol. 2017;35:3105-12.

5. Ma F, Ouyang Q, Li W, Jiang Z, Tong Z, Liu Y, et al. Pyrotinib or lapatinib combined with capecitabine in HER2-positive metastatic breast cancer with prior taxanes, anthracyclines, and/or trastuzumab: a randomized, phase II study. J Clin Oncol. 2019;37:2610-9.

6. Saura C, Oliveira M, Feng YH, Dai MS, Hurvitz SA, Kim SB, et al. Neratinib + capecitabine versus lapatinib + capecitabine in patients with HER2+ metastatic breast cancer previously treated with $\geq 2$ HER2-directed regimens: findings from the multinational, randomized, phase III NALA trial. J Clin Oncol. 2019;37(15 Suppl):1002.

7. Awada A, Colomer R, Inoue K, Bondarenko I, Badwe RA, Demetriou G, et al. Neratinib plus paclitaxel vs trastuzumab plus paclitaxel in previously untreated metastatic ERBB2-positive breast cancer: the NEfERT-T randomized clinical trial. JAMA Oncol. 2016;2:1557-64.

8. Gourd E. Pyrotinib versus lapatinib in HER2-positive breast cancer. Lancet Oncol. 2019;20:e562.

9. Freedman RA, Gelman RS, Anders CK, Melisko ME, Parsons 
HA, Cropp AM, et al. TBCRC 022: a phase II trial of neratinib and capecitabine for patients with human epidermal growth factor receptor 2-positive breast cancer and brain metastases. J Clin Oncol. 2019;37:1081-9.

10. Martin AM, Cagney DN, Catalano PJ, Warren LE, Bellon JR, Punglia RS, et al. Brain metastases in newly diagnosed breast cancer: a population-based study. JAMA Oncol. 2017;3:106977.

11. Costa R, Carneiro BA, Wainwright DA, Santa-Maria CA, Kumthekar P, Chae YK, et al. Developmental therapeutics for patients with breast cancer and central nervous system metastasis: current landscape and future perspectives. Ann Oncol. 2017;28:44-56.

12. Brufsky AM, Mayer M, Rugo HS, Kaufman PA, Tan-Chiu E, Tripathy D, et al. Central nervous system metastases in patients with HER2-positive metastatic breast cancer: incidence, treatment, and survival in patients from registHER. Clin Cancer Res. 2011;17:4834-43.

13. Krop IE, Lin NU, Blackwell K, Guardino E, Huober J, Lu M, et al. Trastuzumab emtansine (T-DM1) versus lapatinib plus capecitabine in patients with HER2-positive metastatic breast cancer and central nervous system metastases: a retrospective, exploratory analysis in EMILIA. Ann Oncol. 2015;26: 113-9.

14. Swain SM, Baselga J, Miles D, Im YH, Quah C, Lee LF, et al. Incidence of central nervous system metastases in patients with HER2-positive metastatic breast cancer treated with pertuzumab, trastuzumab, and docetaxel: results from the randomized phase III study CLEOPATRA. Ann Oncol. 2014;25: 1116-21.

15. Petrelli F, Ghidini M, Lonati V, Tomasello G, Borgonovo K, Ghilardi M, et al. The efficacy of lapatinib and capecitabine in HER-2 positive breast cancer with brain metastases: A systematic review and pooled analysis. Eur J Cancer. 2017;84: 141-8.

16. Kim JM, Miller JA, Kotecha R, Chao ST, Ahluwalia MS, Peereboom DM, et al. Stereotactic radiosurgery with concurrent HER2-directed therapy is associated with improved objective response for breast cancer brain metastasis. Neuro Oncol. 2019;21:659-68. 